\title{
Transport of thermal plasma above the auroral ionosphere in the presence of electrostatic ion-cyclotron turbulence
}

\author{
V. E. Zakharov $^{1}$ and C.-V. Meister ${ }^{2}$ \\ ${ }^{1}$ Kaliningrad State University, Russia \\ ${ }^{2}$ Astrophysical Institute Potsdam, Germany \\ Received: 6 October 1997 / Revised: 4 June 1998 / Accepted: 8 June 1998
}

\begin{abstract}
The electron component of intensive electric currents flowing along the geomagnetic field lines excites turbulence in the thermal magnetospheric plasma. The protons are then scattered by the excited electromagnetic waves, and as a result the plasma is stable. As the electron and ion temperatures of the background plasma are approximately equal each other, here electrostatic ion-cyclotron (EIC) turbulence is considered. In the nonisothermal plasma the ion-acoustic turbulence may occur additionally. The anomalous resistivity of the plasma causes large-scale differences of the electrostatic potential along the magnetic field lines. The presence of these differences provides heating and acceleration of the thermal and energetic auroral plasma. The investigation of the energy and momentum balance of the plasma and waves in the turbulent region is performed numerically, taking the magnetospheric convection and thermal conductivity of the plasma into account. As shown for the quasi-steady state, EIC turbulence may provide differences of the electric potential of $\Delta V \approx 1-$ $10 \mathrm{kV}$ at altitudes of $500<h<10000 \mathrm{~km}$ above the Earth's surface. In the turbulent region, the temperatures of the electrons and protons increase only a few times in comparison with the background values.
\end{abstract}

Key words. Magnetospheric physics (electric fields; plasma waves and instabilities)

\section{Introduction}

Early particle observations by rockets and low altitude satellites suggested that electrons causing the aurora are energized by a quasi-static electric field parallel to the geomagnetic field B (Evans, 1974). The auroral arcs may

Correspondence to: C.-V. Meister be present because the parallel electric fields accelerate the auroral electrons downwards (Chiu and Cornwall, 1980). Generally this is still believed to be true. However, it is now realized that the wave-particle interactions are important for particle energization in the auroral region. The electric component of these waves, which is parallel to $\mathbf{B}$, modulates the field-aligned fluxes of electrons at the wave frequency. Gustafsson et al. (1990) reported the extremely low frequency (ELF) waves below the proton cyclotron frequency in the Viking satellite electric field data. In the case of electromagnetic ion cyclotron (EMIC) waves, the modulation effect mentioned already has been detected by sounding rockets (Temerin et al., 1993; Lund and LaBelle, 1997).

The EMIC waves were observed by Freja satellite in a region of inverted $\mathrm{V}$-type electron precipitation (Erlandson et al., 1994). Simultaneous Freja observations of precipitating $\mathrm{keV}$ electrons and electromagnetic emissions around half the local proton gyrofrequency were analyzed by Oscarsson et al. (1997). They estimated that the interaction between the EMIC waves and the electrons is resonant at altitudes of several thousands of kilometers above the auroral ionosphere.

Waves also may be essential for the formation of a quasi-static difference of the electric potential. Observations, including those obtained by polar orbiting satellites, have shown that the auroral acceleration region is typically located between magnetic latitudes of $65^{\circ}$ and $75^{\circ}$, and corresponds to a potential difference of $1-$ $10 \mathrm{kV}$. The potential difference is usually located at altitudes between 2000 and $15000 \mathrm{~km}$ (Reiff et al., 1988, 1993). At altitudes of $1-2 R_{E}$, where $R_{E}$ is the Earth's radius, perpendicular spatial scales of about $100 \mathrm{~km}$ are most common for the parallel electric fields (Gorney, 1984). However, finer scales have also been observed, embedded in the larger features. Thus, the Freja satellite locally detected strong electric spikes of about $100 \mathrm{mV} / \mathrm{m}$ (Chust et al., 1998).

Kindel and Kennel (1971) emphasized the local generation of microinstabilities by intensive field-aligned 
currents. The microinstabilities cause the anomalous resistivity in the plasma and the appearance of the electric fields $E_{\|}$parallel to the geomagnetic field (Lysak and Dum, 1983). The resistance would be due to waveparticle interactions in the turbulent plasma.

Kindel and Kennel (1971) suggested that the electrostatic ion cyclotron (EIC) waves could be produced by field-aligned currents. These emissions have spectral peaks above the ion gyrofrequencies and sometimes at higher harmonics. The EIC waves are usually observed on auroral field lines (André et al., 1987). Typical amplitudes of the electric field are a few $\mathrm{mV} / \mathrm{m}$, but can be larger, and the wavelengths are of the order of the ion gyroradius. The importance of EIC waves for the formation of the auroral potential difference is still uncertain (André 1997).

Thus, in this work, the influence of EIC turbulence on the transport of the plasma of the auroral magnetosphere is investigated using magnetohydrodynamics. In Sect. 2, the model equations are obtained to describe this transport. In Sect. 3, the model description of the EIC turbulence is presented. In Sect. 4, anomalous heating effects, the thermal conductivity, and the electric fields parallel to the geomagnetic field in the region of quasi-stationary EIC turbulence are studied. This is done numerically in dependence on the intensity of fieldaligned currents, the thickness of the turbulent region perpendicular to the geomagnetic field, and on the intensity of the EIC waves in the plasma.

\section{Transport equations for thermal plasma in the case of developed turbulence}

We consider the plasma region located at altitudes of $h_{b}<h<h_{m}$ in the auroral ionosphere and magnetosphere where $h_{b}=500 \mathrm{~km}$ and $h_{m}=10000 \mathrm{~km}$. There, the magnetic field is assumed to be dipolar. The spherical components of the magnetic induction $\mathbf{B}$ may be written as $B_{r}=-2 B_{e} \cos \theta / r^{3}, B_{\theta}=-B_{e} \sin \theta / r^{3}$, and $B_{\lambda}=0$ where $r$ is the geocentric distance expressed in units of the Earth's radius $R_{E}, \theta$ and $\lambda$ are the geomagnetic colatitude and longitude, respectively, and $B_{e}=3.1 \cdot 10^{-5} \mathrm{~T}$. The dipolar coordinate system is used (Fatkullin and Sitnov, 1972). The dipolar coordinates $\{\varphi, \beta, \lambda\}$ are related to the spherical ones $\{r, \theta, \lambda\}$ using $1 / \sin ^{2} \varphi=r / \sin ^{2} \theta, \beta=\cos \theta / r^{2}$. The magnetic field lines form the $\beta$-set of coordinate lines. The coordinates $\varphi$ and $\lambda$ change their values only along axes perpendicular to $\mathbf{B}$. Thus, a use of the coordinates $\{\varphi, \beta, \lambda\}$ instead of $\{r, \theta, \lambda\}$ is warranted at altitudes of $h_{b}<h<h_{m}$ in the auroral magnetosphere. On the Earth's surface where $r=1$, the coordinate $\varphi$ equals to the geomagnetic colatitude $\theta$.

Let us denote $x^{1}=\varphi, x^{2}=\beta$, and $x^{3}=\lambda$, and $g_{i k}(i, k=1,2,3)$ is the metric tensor of the coordinate system. The components of the metric tensor are as follows: $g_{i k}=0$ for $i \neq k, g_{11}=4 r^{3} \cos ^{2} \varphi / \delta, g_{22}=r^{6} / \delta$, and $g_{33}=r^{3} \sin ^{2} \varphi$ where $\delta=4-3 r \sin ^{2} \varphi$.

We suppose the thermal magnetospheric plasma to consist of protons and electrons. A magnetic flux tube of the nightside auroral magnetosphere is chosen. Let $\Phi$ be the magnetic flux inside the tube, and $j_{2}$ is the density of the electric current flowing in this tube along the magnetic field. The current $j_{2}$ is closed by the electric currents flowing perpendicular to the magnetic field, both in the ionosphere, and in the plasma sheet of the magnetosphere. We suppose that such a closing takes place outside the altitude range mentioned. A proper treatment of the ionospheric and magnetospheric physics that would allow to explain the closing is beyond the scope of this work. We suppose that the current $j_{2}$ is sufficiently intensive to provide the developed EIC turbulence in the thermal plasma located inside the given flux tube. The turbulent plasma is immersed into the background thermal plasma.

Now we will present the system of model equations to describe the behaviour of the thermal plasma in the region of quasi-stationary turbulence. To do this, we use the magnetohydrodynamic approximation (Goldstone and Rutherford, 1995).

Let $n_{j}, T_{j}$, and $\mathbf{v}_{j}$ be the density, temperature, and mean velocity per particle, respectively, taken inside a chosen flux tube, and $j=1$ for protons $(j=2$ for electrons). It is supposed that the thermal plasma obeys the condition of quasi-neutrality so that $n_{1}=n_{2}$. Only a cold plasma is considered, in which the pressure of the plasma is much less than the pressure of the magnetic field. In this case, the component of the mean velocity perpendicular to $\mathbf{B}$ may be approximated by the velocity of the electric drift per particle as $\mathbf{v}_{E}=\mathbf{E} \times \mathbf{B} / B^{2}$ where $\mathbf{E}$ is the strength of the electric field. The velocity $\mathbf{v}_{E}$ is present due to the magnetospheric convection. The component of $\mathbf{v}_{j}$ parallel to $\mathbf{B}$ equals $\mathbf{v}_{j 2}=\mathbf{v}_{j}-\mathbf{v}_{E}$. The inertial force per particle equals $-m_{j}\left(\mathbf{v}_{j} \nabla\right) \mathbf{v}_{j}, m_{j}$ is the mass per particle. According to our estimates, $-(\mathbf{B} / B) m_{j}\left(\mathbf{v}_{j} \nabla\right) \mathbf{v}_{j} \approx-(\mathbf{B} / B) m_{j}\left(\mathbf{v}_{j} \nabla\right) \mathbf{v}_{j 2}$.

Further, $\mathbf{R}_{t j}$ and $\mathbf{R}_{u j}$ designate the thermoforce and the friction force, respectively, caused by scattering of charged particles in the turbulent plasma. Assuming developed turbulence, we suppose that the total difference of the electric potential $V$ along $\mathbf{B}$ over the turbulent region given obeys $\Delta V>0.1 \mathrm{kV}$. In this case, $\Delta V \gg k_{B} T_{j} / e$ where $k_{B}$ and $e$ are the Boltzmann constant and elementary charge, respectively. On the other hand, $\quad \mathbf{R}_{t 1}=-\mathbf{R}_{t 2}$, and $\left|\mathbf{R}_{t 2} \mathbf{B} / B\right| \sim n_{2} k_{B}$ $\left|\left(1 / \sqrt{g_{22}}\right) \partial T_{2} / \partial x^{2}\right|$ (Goldstone and Rutherford, 1995). Then, we neglect both the effects of $\mathbf{R}_{t j}$ and of the pressure gradients $-\nabla\left(n_{j} k_{B} T_{j}\right)$ in the plasma in comparison with those caused by the electric force $Z_{j} e n_{j} \mathbf{E}$ where $Z_{1}=1$ and $Z_{2}=-1$.

In the present approximations, the continuity equation of the plasma located in the given volume of flux tube may be given in the integro-differential form as

$\int_{L} \sqrt{g_{22}} n_{j} \frac{\mathbf{B}}{B} \times \mathbf{v}_{j} \quad \mathrm{~d} \mathbf{L}=0$

where $L$ is the contour bounding the surface $S$. The choice of the surface $S$ obeys the following relation for the magnetic flux $\Phi=\int_{S} \mathbf{B} \mathrm{d} \mathbf{S}$, and $\mathrm{d} \mathbf{S}=(\mathbf{B} / B)$ $\sqrt{g_{11} g_{33}} \mathrm{~d} x^{1} \mathrm{~d} x^{3}$. 
Under the same conditions, we obtain the following equation describing the momentum balance in the plasma along $\mathbf{B}$

$$
\begin{aligned}
\int_{S} \sqrt{g_{22}}\left(Z_{j} e n_{j} \mathbf{E}+\mathbf{R}_{u j}\right) \frac{\mathbf{B}}{B} \mathrm{~d} S \\
-\int_{L} \sqrt{g_{22}} m_{j} n_{j} v_{j 2} \frac{\mathbf{B}}{B} \times \mathbf{v}_{j} \quad \mathrm{~d} \mathbf{L}=0 .
\end{aligned}
$$

The energy equation of the thermal plasma may be written approximately as

$$
\begin{aligned}
\frac{\partial}{\partial x^{2}}\left(\int_{S} q_{j} \mathrm{~d} S\right)= & \int_{S} \sqrt{g_{22}}\left(Z_{j} e n_{j} \mathbf{v}_{j} \mathbf{E}+\gamma_{j} W\right) \mathrm{d} S \\
& -\int_{L} \sqrt{g_{22}} \frac{\mathbf{B}}{B} \times \mathbf{\Pi}_{j \perp} \mathrm{d} \mathbf{L},
\end{aligned}
$$

where $\gamma_{1}$ is the growth rate of the EIC waves scattering the electrons, $\gamma_{2}$ is the damping rate of the EIC waves scattering the protons, $W$ is the energy of the waves per unit of volume, $q_{j}$ is the thermal flux density caused by thermal conductivity of the plasma only, $q_{j}=-5 n_{j} k_{B} T_{j} /\left(2 m_{j} v_{j} \sqrt{g_{22}}\right) \partial\left(k_{B} T_{j}\right) / \partial x^{2}$, and $v_{j}$ is the collision frequency of the particles scattered by the EIC waves, $\boldsymbol{\Pi}_{j \perp}$ is the component of the total energy flux density of the particles taken perpendicular to $\mathbf{B}$ so that $\boldsymbol{\Pi}_{j \perp}=n_{j} \mathbf{v}_{E}\left(5 k_{B} T_{j}+m_{j} v_{j}^{2}\right) / 2$.

We suppose that $r_{\perp B} \gg r_{\perp}$ where $r_{\perp B}$ and $r_{\perp}$ are the spatial scales of the magnetic field and of the given flux tube taken across $\mathbf{B}$. By definition $\pi r_{\perp}^{2}=\Phi / \mathbf{B}$, so that $r_{\perp}=\sqrt{\Phi /(\pi \mathbf{B})}$ for the flux tube given already. This allows us to simplify Eqs. (1), (2), and (3) further. For the parameters inside the flux tube we use the effective values. The set of parameters of the background plasma will be denoted by the superscript "**". In our model, these effective values may depend on $x^{2}$, but do not depend on $x^{1}$ and $x^{3}$ when the flux tube is given. Using the given approximations, we reduce Eq. (1) to $\sqrt{g_{22}} \Delta V_{\perp}\left(n_{j}-n_{j}^{*}\right) / B=0$ where $\Delta V_{\perp}$ is the total difference of the electric potential across the given flux tube that is supposed to be caused by the magnetospheric convection and constant along the flux tube. When $\Delta V_{\perp} \neq 0$, the latter equation may be replaced by the following one

$n_{j}=n_{j}^{*}=n_{e}$,

where $n_{e}$ is the electron density of the background thermal plasma, $n_{e}=n_{2}^{*}$.

Taking the maxwellian equation $\nabla \mathbf{B}=0$ into account and employing similar transformations as in the case of Eq. (4), we reduce Eqs. (2) and (3) to

$Z_{j} e n_{e} E_{2}+R_{u j 2}=C m_{j} n_{e}\left(v_{j 2}-v_{j 2}^{*}\right)$,

where $C=\Delta V_{\perp} / \Phi$, and

$$
\begin{gathered}
-B \frac{\partial}{\partial s_{2}}\left(\frac{5}{2 B} \frac{n_{e} k_{B} T_{j}}{m_{j} v_{j}} \frac{\partial\left(k_{B} T_{j}\right)}{\partial s_{2}}\right)=Z_{j} e n_{e} v_{j 2} E_{2}+\gamma_{j} W \\
-\frac{C m_{j} n_{e}}{2}\left(v_{j 2}^{2}-\left(v_{j 2}^{*}\right)^{2}\right)-\frac{5 C n_{e} k_{B}}{2}\left(T_{j}-T_{j}^{*}\right),
\end{gathered}
$$

where $\mathrm{d} s_{2}$ is the element of length along $\mathbf{B}$, and $\mathrm{d} s_{2}=\sqrt{g_{22}} \mathrm{~d} x^{2}$.
Taking the presence of the intensive current $j_{2}$ in the turbulent region into account, we neglect the velocity $v_{j 2}^{*}$ in comparison with $v_{j 2}$. According to the third law of Newton, we have $R_{u 12}=-R_{u 22}$. Then, substituting $Z_{1}=-Z_{2}=1$ Eq. (5) can be transformed into

$v_{12}=-\frac{m_{2}}{m_{1}} v_{22}$,

$E_{2}=\frac{1}{e n_{e}}\left(R_{u 22}-C m_{2} n_{e} v_{22}\right)$.

Substituting Eq. (8) into Eq. (6), we obtain under the mentioned conditions

$$
\begin{aligned}
&-B \frac{\partial}{\partial s_{2}}\left(\frac{5}{2 B} \frac{n_{e} k_{B} T_{j}}{m_{j} v_{j}} \frac{\partial\left(k_{B} T_{j}\right)}{\partial s_{2}}\right)=-v_{j 2} R_{u j 2}+\gamma_{j} W \\
&+\frac{C m_{j} n_{e}}{2} v_{j 2}^{2}-\frac{5 C n_{e} k_{B}}{2}\left(T_{j}-T_{j}^{*}\right) .
\end{aligned}
$$

Neglecting $v_{12}$ in comparison with $v_{22}$ and considering the approximations given, we obtain

$$
v_{22}=-\frac{j_{2 \mid b}}{e n_{e}} \frac{B}{B_{b}}
$$

where $j_{2 \mid b}$ is the density of the electric current flowing in the given flux tube at the altitude $h=h_{b}$ at which $B=B_{b}$.

Thus, we have obtained the system of equations Eqs. (4) and (7)-(10) to calculate the quantities $n_{j}, v_{j 2}, E_{2}$, and $T_{j}$ in the turbulent region.

\section{Model description of the EIC turbulence}

We investigate the anomalous transport of thermal plasma in the case of developed EIC turbulence.

Let $\omega_{c}$ be the gyrofrequency of a proton, $\omega_{c}=e B / m_{1}, k$ be the wave number, and $v_{T_{j}}$ - the thermal velocity of a particle, $v_{T_{j}}=\sqrt{2 k_{B} T_{j} / m_{j}}$. When $\omega_{c} \sim k v_{T 1}$, the dispersion equation of the EIC waves may be expressed in approximate form as follows (Lominadze and Stepanov, 1964)

$1+\frac{T_{1}}{T_{2}}=f$,

where

$f=e^{-\mu}\left(I_{0}(\mu)+2 \sum_{n=1}^{\infty} \frac{\omega^{2} I_{n}(\mu)}{\omega^{2}-n^{2} \omega_{c}^{2}}\right)$,

and $I_{n}$ is the modified Bessel function specified by its order $n, n=0,1,2 \ldots, \omega$ is the frequency of oscillations, $\mu=(1 / 2)\left(k v_{T 1} \sin \xi / \omega_{c}\right)^{2}, \xi$ is the angle between $\mathbf{k}$ and $\mathbf{B}$, and $\mathbf{k}$ is the wave vector.

As known, the interaction between EIC waves and the charged particles may develop the resonant properties. This interaction is most effective when $\omega \sim n \omega_{c}$ where $n=1,2,3 \ldots$ According to Lominadze and Stepanov (1964), the ground mode $(n=1)$ of the EIC oscillations may become unstable at a lower current density than the other modes $(n>1)$. For that reason, we consider only the ground mode. 
The analysis of Eq. (11) shows that the frequency $\omega$ obeys the following inequalities: $\omega_{c}<\omega \leq \omega_{b}$ where $\omega_{b}$ is the maximum value of $\omega$. Differentiating Eq. (11) for given ratio of $T_{1} / T_{2}$, we obtain $(\partial f / \partial \mu)_{\omega} \mathrm{d} \mu+$ $(\partial f / \partial \omega)_{\mu} \mathrm{d} \omega=0$. As $\mathrm{d} \omega / \mathrm{d} \mu=0$ for $\omega=\omega_{b}$, we obtain $(\partial f / \partial \mu)_{\omega=\omega_{b}}=0$. We combine the latter equation and Eq. (11) into one system. Integrating this system numerically, we calculate both the values of $\omega_{b}$ and $\mu_{b}$ where $\mu=\mu_{b}$ for $\omega=\omega_{b}$. For the given ratio $T_{1} / T_{2}, \mu$ may be calculated numerically in dependence on $\omega$ integrating Eq. (11). As a result, for $0<\mu \leq \mu_{b}\left(\mu \geq \mu_{b}\right)$, we propose an approximate function $\mu=\mu_{1}(\omega)(\mu=$ $\left.\mu_{2}(\omega)\right)$ for $\omega_{c}<\omega \leq \omega_{b}$.

We describe the spectral components of the damping (growth) rate of the EIC waves according to Lominadze and Stepanov (1964) by

$\gamma_{1 \ell \omega}=\sum_{m=-\infty}^{\infty} \gamma_{1 m \ell \omega}$

where $\ell=1,2$ for $\mu=\mu_{1}(\omega)$ and $\mu=\mu_{2}(\omega)$, respectively,

$\gamma_{1 m \ell \omega}=\sqrt{\frac{\pi}{32}} \frac{\omega_{c}}{\sqrt{\mu_{\ell}} \cos \xi_{\ell} F_{\ell}} e^{-\mu_{\ell}} I_{m}\left(\mu_{\ell}\right) e^{-z_{m \ell}^{2}}$,

$z_{m_{\ell}}=\frac{\omega-m \omega_{c}}{k_{\ell} v_{T 1} \cos \xi_{\ell}}$,

$F_{\ell}=e^{-\mu_{\ell}} \sum_{n=1}^{\infty} \frac{\omega_{c}^{4} n^{2} I_{n}\left(\mu_{\ell}\right)}{\left(\omega^{2}-n^{2} \omega_{c}^{2}\right)^{2}}$,

and

$\gamma_{2 \ell \omega}=\left(1-\frac{k_{\ell} v_{22} \cos \xi_{\ell}}{\omega}\right) \sqrt{\frac{\pi m_{2} T_{1}^{3}}{32 m_{1} T_{2}^{3}}} \frac{\omega_{c}}{\sqrt{\mu_{\ell}} \cos \xi_{\ell} F_{\ell}}$.

To calculate $\gamma_{j \ell \omega}$, we, at first, calculate the component of $\mathbf{k}_{\ell}$ parallel to $\mathbf{B}$ which is equal to $k_{\ell} \cos \xi_{\ell}$. To this purpose, we employ the equation $\sum_{j=1}^{2} \gamma_{j \ell \omega}=0$ describing the balance between growth and damping of the wave energy. Using Eqs. (12) and (13), we reduce the latter equation to

$$
\begin{aligned}
&(1\left.-\frac{k_{\ell} v_{22} \cos \xi_{\ell}}{\omega}\right) \sqrt{\frac{m_{2} T_{1}^{3}}{m_{1} T_{2}^{3}}} \\
&+\sum_{m=-\infty}^{\infty} e^{-\mu_{\ell}} I_{m}\left(\mu_{\ell}\right) e^{-z_{m \ell}^{2}}=0
\end{aligned}
$$

which is integrated numerically to calculate the product $k_{\ell} \cos \xi_{\ell}$.

For lack of data, we use a crude approximation to give the spectrum $W_{\ell \omega}$ of the wave energy per unit of volume. We suppose that both $W_{\ell \omega}$ and $\gamma_{2 \ell \omega}$ take the maximum values at the same frequency $\omega_{\ell}$. This frequency may be estimated since $\gamma_{2 \ell \omega}$ is already calculated for $\omega_{c}<\omega \leq \omega_{b}$. It is important to estimate how much the results of our numerical calculations of the plasma parameters in the turbulent region depend on a choice of the spectrum $W_{\ell \omega}$. To this purpose, we perform the calculations for two spectra. The first of them is given as

$W_{\ell \omega}=A_{\ell}\left(\omega-\omega_{c}\right)^{a_{\ell}}\left(\omega_{b}-\omega\right)^{b_{\ell}}$,

where $b_{\ell}$ is a model parameter $\left(b_{\ell}>0\right), a_{\ell}=\left(\omega_{\ell}-\omega_{c}\right) b_{\ell} /$ $\left(\omega_{b}-\omega_{\ell}\right), A_{\ell}=W_{\ell} / \int_{\omega_{c}}^{\omega_{b}}\left(\omega-\omega_{c}\right)^{a_{\ell}}\left(\omega_{b}-\omega\right)^{b_{\ell}} \mathrm{d} \omega$ due to the normalization condition $W=\sum_{\ell=1}^{2} \int_{\omega_{c}}^{\omega_{b}} W_{\ell \omega} \mathrm{d} \omega=$ $\sum_{\ell=1}^{2} W_{\ell}$, and $W_{1}=W_{2}$.

For the same value of $W_{\ell}$ as used in Eq. (15), we give the second spectrum to be equal to

$W_{\ell \omega}=W_{\ell} \delta\left(\omega-\omega_{\ell}\right)$,

where $\delta$ is the Dirac delta-function, and $\int_{\omega_{c}}^{\omega_{b}} \delta(\omega-$ $\left.\omega_{\ell}\right) \mathrm{d} \omega=1$ as $\omega_{c}<\omega_{\ell}<\omega_{b}$.

Using both spectra in our calculations, we have arrived at the following conclusion. With respect to the order of magnitude, our numerical results for the plasma parameters are the same for both these spectra when the values of $W$ are the same. For that reason, in Sect. 4, we shall present only the results of the calculations obtained using Eq. (16).

The turbulence intensity may be estimated by the ratio $W /\left(n k_{B} T_{2}\right)$. We use a crude approximation to give the behaviour of $W /\left(n k_{B} T_{2}\right)$ along $\mathbf{B}$ in the turbulent region. For the case of developed turbulence, we suppose that $W /\left(n k_{B} T_{2}\right)$ increases while $\gamma_{2}$ increases. On the other hand, the analysis shows that $\gamma_{2}$ decreases while $v_{22} / v_{T 2}$ and $T_{2} / T_{1}$ decrease. Let us give the value of the ratio $\mathrm{W} /\left(n k_{B} T_{2}\right)$ as a model parameter, for instance, at $h=h_{m}$. Then, we estimate the behaviour of this ratio along $\mathbf{B}$ in the turbulent region using the following model relation $W /\left(n k_{B} T_{2}\right) \sim\left(v_{22} / v_{T 2}\right)\left(T_{2} / T_{1}\right)$.

Following Artsymovich and Sagdeev (1979), we obtain

$\left\{\begin{array}{l}\gamma_{j} W=\sum_{\ell=1}^{2} \int_{\omega_{c}}^{\omega_{b}} \gamma_{j \ell \omega} W_{\ell \omega} \mathrm{d} \omega \\ R_{u j 2}=-m_{j} n_{j} v_{j} v_{j 2}=\sum_{\ell=1}^{2} \int_{\omega_{c}}^{\omega_{b}} \gamma_{j \ell \omega} W_{\ell \omega} \frac{k_{\ell} \cos \xi_{\ell}}{\omega} \mathrm{d} \omega .\end{array}\right.$

When the quantities $n_{j}, v_{j 2}$, and $T_{j}$ are given, Eq. (17) allows us to calculate the quantities $\gamma_{j} W, R_{u j 2}$, and $m_{j} v_{j}$ in the turbulent region using the algorithm presented. Combining Eqs. (4) and (7)-(17), we obtain the system of non-linear equations describing the thermal plasma and the EIC waves.

\section{Discussion of the results of the numerical calculations}

For the thermal background plasma, we give the patterns of $n_{e}$ and $T_{j}^{*}$. To this purpose, we use the profiles $n_{e}(h)$ and $T_{j}^{*}(h)$ obtained by averaging of the available satellite data over the nightside auroral zone (Liperovsky and Pudovkin, 1983). In our calculations, we give the coordinate $\varphi=15^{\circ}$. Figure 1 shows the patterns of $n_{c}(h)$ and $T_{j}^{*}(h)$ mentioned already.

It is convenient to rewrite the expression for the model parameter $C$ as $C=\Delta V_{\perp} / \Phi \sim 2 E_{\perp} r_{\perp} /\left(B \pi r_{\perp}^{2}\right)=$ $2 v_{E} /\left(\pi r_{\perp}\right)$ where $E_{\perp}$ is the strength of the electric field of the magnetospheric convection. Giving the values of $E_{\perp}$ 

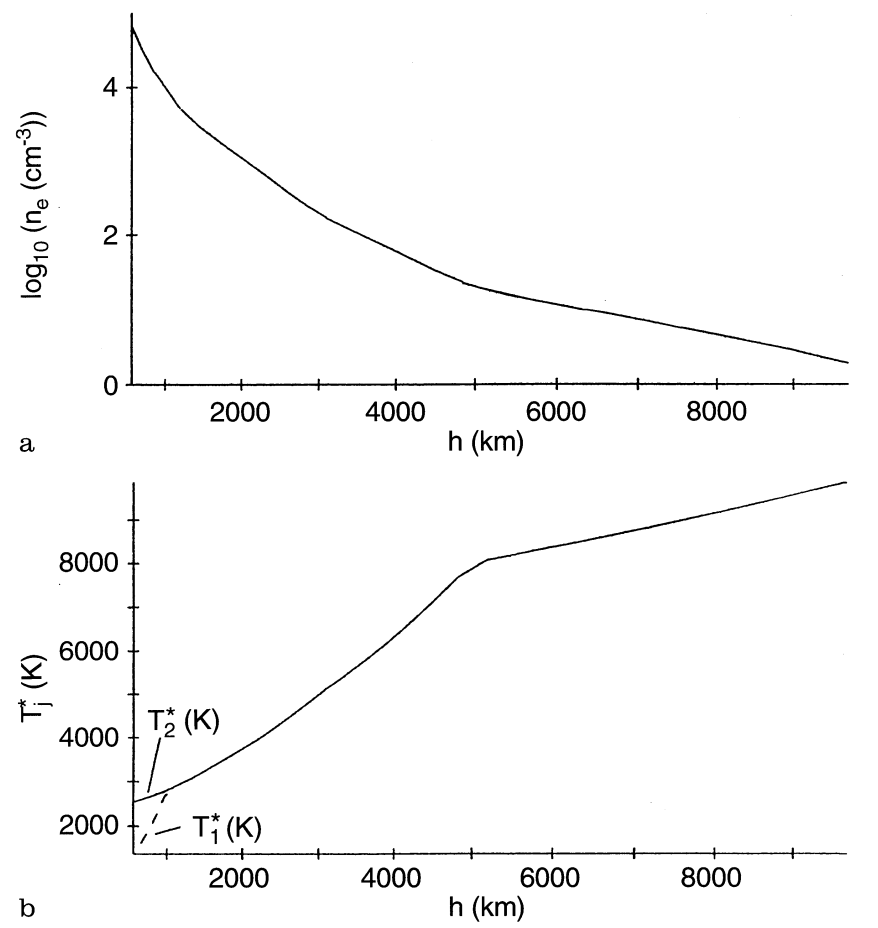

Fig. 1. a Electron density $n_{e}$ and $\mathbf{b}$ temperatures $T_{j}^{*}$ of protons $(j=1)$ and electrons $(j=2)$ versus altitude $h$ obtained for the background plasma located above the nightside auroral ionosphere by averaging of experimental data (Liperovsky and Pudovkin, 1983)

and $r_{\perp}$ at $h=h_{b}$, we calculate the parameter $C$. As known, the intensity both of the ionospheric and magnetospheric convection depends strongly on geomagnetic activity. According to experimental data and the model estimates (Foster et al. 1986; Heppner and Maynard, 1987; Holt et al. 1987; Senior et al. 1990) the maximum values of $E_{\perp}$ may be about $10-100 \mathrm{mV} / \mathrm{m}$ on the nightside of the auroral ionosphere. The intensity of the electric currents flowing along $\mathbf{B}$ above the auroral ionosphere also depends strongly on geomagnetic activity. According to experimental data (Iijima and Potemra, 1978), the maximum values of $j_{\|}=j_{2}$ may be of the order of $10^{-6}-10^{-5} \mathrm{~A} / \mathrm{m}^{2}$. Hudson et al. (1978) and Torbert and Mozer (1978), using satellite data, concluded that the intensity of the developed electrostatic turbulence in the auroral magnetosphere may be as large as $W /\left(n_{2} k_{B} T_{2}\right) \sim 10^{-4}-10^{-3}$.

Our purpose is to investigate the behaviour of the turbulent plasma for the different scales $r_{\perp}$ at which intensive parallel currents may occur. This will be done in dependence on the intensity of the magnetospheric convection and parallel currents.

Let us discuss the numerical algorithm employed to integrate the system of Eqs. (4) and (7)-(17) and the results of our numerical calculations. In the most simple case, when the turbulence is weak or (and) the parameter $C$ takes sufficiently great values, the effects of turbulence may be neglected during the calculations. The more intensive the convection, and the narrower the flux tube in which the intensive parallel current flows, the greater the values of $C$. In this case, we may neglect the first term on the right side of Eq. (8). For the same reason, the term on the left side and the first and second terms on the right side of Eq. (9) also may be neglected. As a result, Eqs. (8) and (9) may be reduced to

$$
\begin{aligned}
& E_{2}=-\frac{C}{e} m_{2} v_{22}, \\
& k_{B} T_{j}=k_{B} T_{j}^{*}+\frac{m_{j}}{5} v_{j 2}^{2} .
\end{aligned}
$$

From Eqs. (4), (7), (10), (18), and (19), it follows: the mean velocity of a proton is negligible in comparison with the electron velocity. The parallel current is carried mainly by electrons. The more intensive the current flows in the flux tube the greater the values of the mean electron velocity and electron temperature. But the temperature of the protons in the turbulent region is only slightly greater than their background temperature. While the parallel current becomes more intensive, the ratio $T_{1} / T_{2}$ takes fewer values. This fact may result into the excitation of ion-acoustic turbulence in the given flux tube. Under the conditions given strong inertial forces act on both the electrons and protons along $\mathbf{B}$ in the same flux tube. According to Eq. (18), these forces are balanced by the electric force acting along $\mathbf{B}$. Thus, the difference of the electric potential parallel to $\mathbf{B}$ in the flux tube is mainly due to the action of the inertial force, and not to the effects of anomalous resistivity.

The analysis given above is important as it considers that first intensive currents may appear in narrow flux tubes. Then, with the development of turbulence and the strengthening of the external parallel current source, the large-scale structure of these currents may occur. As the region of turbulence increases, a smaller parameter $C$ has to be considered, and the effects of anomalous resistivity become more important in Eqs. (8) and (9). Thus, the equations Eqs. (18) and (19) are not valid any more.

We use the iteration algorithm to integrate the system of Eqs. (4) and (7)-(10) numerically. In our model, the iterations are used to calculate the temperatures of the protons and electrons self-consistently with the parameters of the EIC waves described in Sect. 3. To this purpose, the method of successive approximations is used (Kalitkin, 1978). To perform the iterations, we add the following term on the right side of Eq. (9): $\partial\left(3 n_{e} k_{B} T_{j} / 2\right) / \partial t$ where $t$ is the parameter of the iterations expressed in units of time. Thus, we replace Eq. (9) by partial differential equations. We perform the difference approximation of the latter equations (Samarsky and Nikolaev, 1978). To integrate the obtained difference equations, we use the sweep method (Samarsky and Nikolaev, 1978). The temperatures of the protons and electrons are given at $h=h_{b}$ and $h=h_{m}$. They are chosen to be close to their background values. The performing of the iterations is ceased when the term $\partial\left(3 n_{e} k_{B} T_{j} / 2\right) / \partial t$ becomes negligible. It should be noted that the method of successive approximations is also used to integrate Eqs. (11) and (14). As was already noted, the interaction between the charged particles and EIC waves is most effective when $\omega \sim \omega_{c}$. For that 

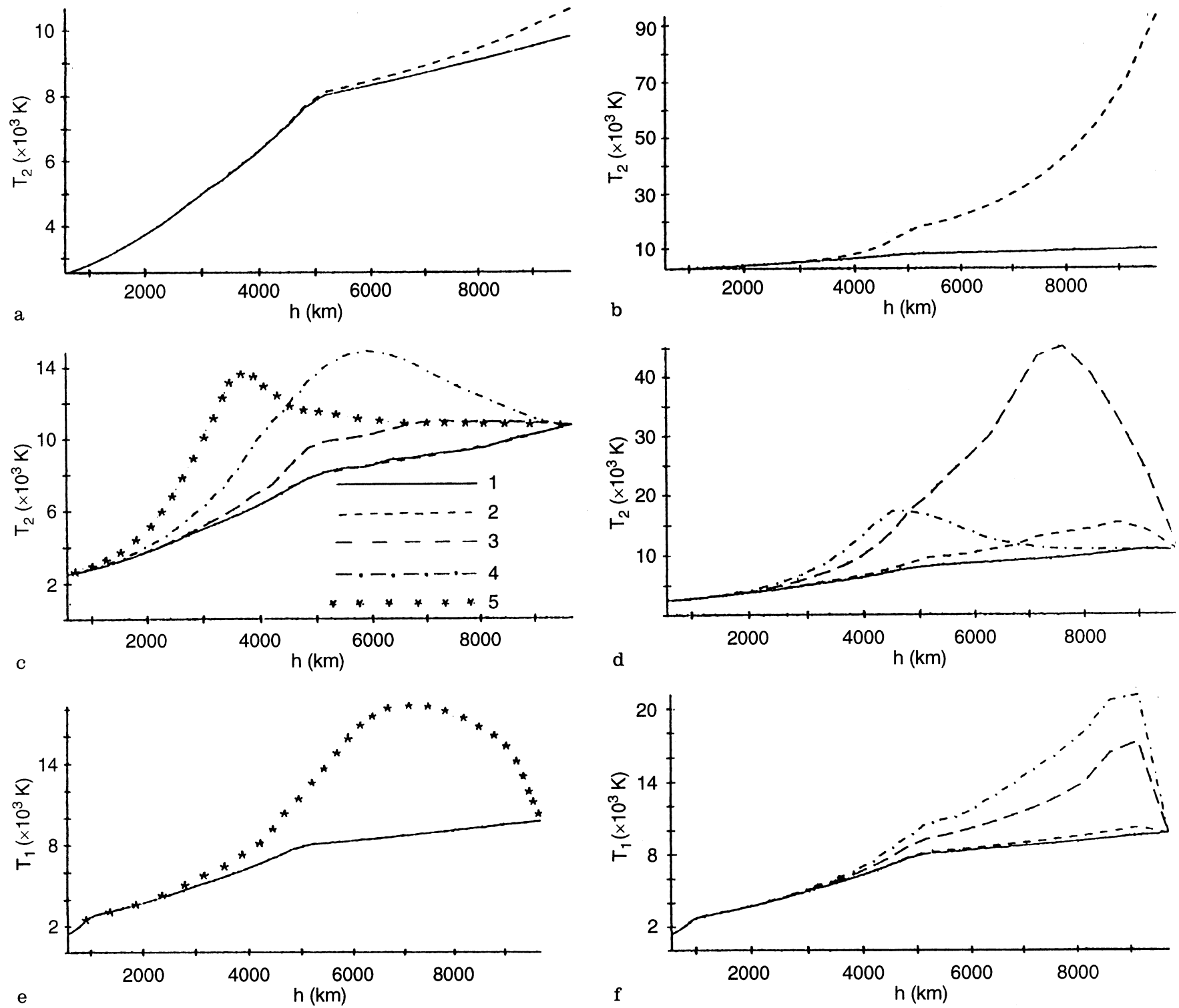

Fig. 2a-f. Temperatures $T_{j}$ of protons $(j=1)$ and electrons $(j=2)$ versus altitude $h$ calculated for the turbulent plasma located in the magnetic flux tube given in the nightside auroral magnetosphere. The maximum value of the ratio of turbulent wave energy to thermal electron energy $W /\left(n_{2} k_{B} T_{2}\right)$ at $h=h_{m}$ equals a, b $10^{-2}, \mathbf{d}, \mathbf{f} 10^{-3}, \mathbf{c}, \mathbf{e}$ $10^{-4}$. Besides, in $\mathbf{a}, \mathbf{b}$, it is $C=1.5 \cdot 10^{2} \mathrm{~s}^{-1}$, and the density of the

parallel electric current amounts to a $j_{2}=10^{-6} \mathrm{~A} / \mathrm{m}^{2}, \quad$ b $j_{2}=10^{-5} \mathrm{~A} / \mathrm{m}^{2}$. Background electron temperature $T_{2}^{*}$, solid curve, electron temperature in turbulent region $T_{2}$, dashed curve. In c-f only the temperatures in the turbulent region are shown, $j_{2}=10^{-6} \mathrm{~A} / \mathrm{m}^{2}$, and $C$ equals (1) $1.5 \mathrm{~s}^{-1}$, (2) $1.5 \cdot 10^{-1} \mathrm{~s}^{-1}, \quad$ (3) $1.5 \cdot 10^{-2} \mathrm{~s}^{-1}$, (4) $1.5 \cdot 10^{-3} \mathrm{~s}^{-1},(5) 1.5 \cdot 10^{-4} \mathrm{~s}^{-1}$

value of $C$. However, different current densities are considered. Figure $2 \mathrm{a}, \mathrm{b}$ is obtained for the case when the density of the parallel current equals $j_{2}=1 \cdot 10^{-6}\left(1 \cdot 10^{-5}\right) \mathrm{A} / \mathrm{m}^{2}$. Under such conditions, the effects of turbulence are negligible and Eqs. (18) and (19) are valid for $h_{b}<h<h_{m}$ excluding the lower altitudes of this region when $W /\left(n_{2} k_{B} T_{2}\right) \leq 1 \cdot 10^{-3}$. To stress on the effects of turbulence, we give the maximum value of $W /\left(n_{2} k_{B} T_{2}\right)$ as equal to $1 \cdot 10^{-2}$ at $h=h_{m}$. The calculated temperature of protons corresponds approximately to that presented in Fig. 1. In contrast in Fig. 2a,b, the difference between the electron temperatures $T_{2}$ (the dashed curves) and $T_{2}^{*}$ (the solid curves) increases 

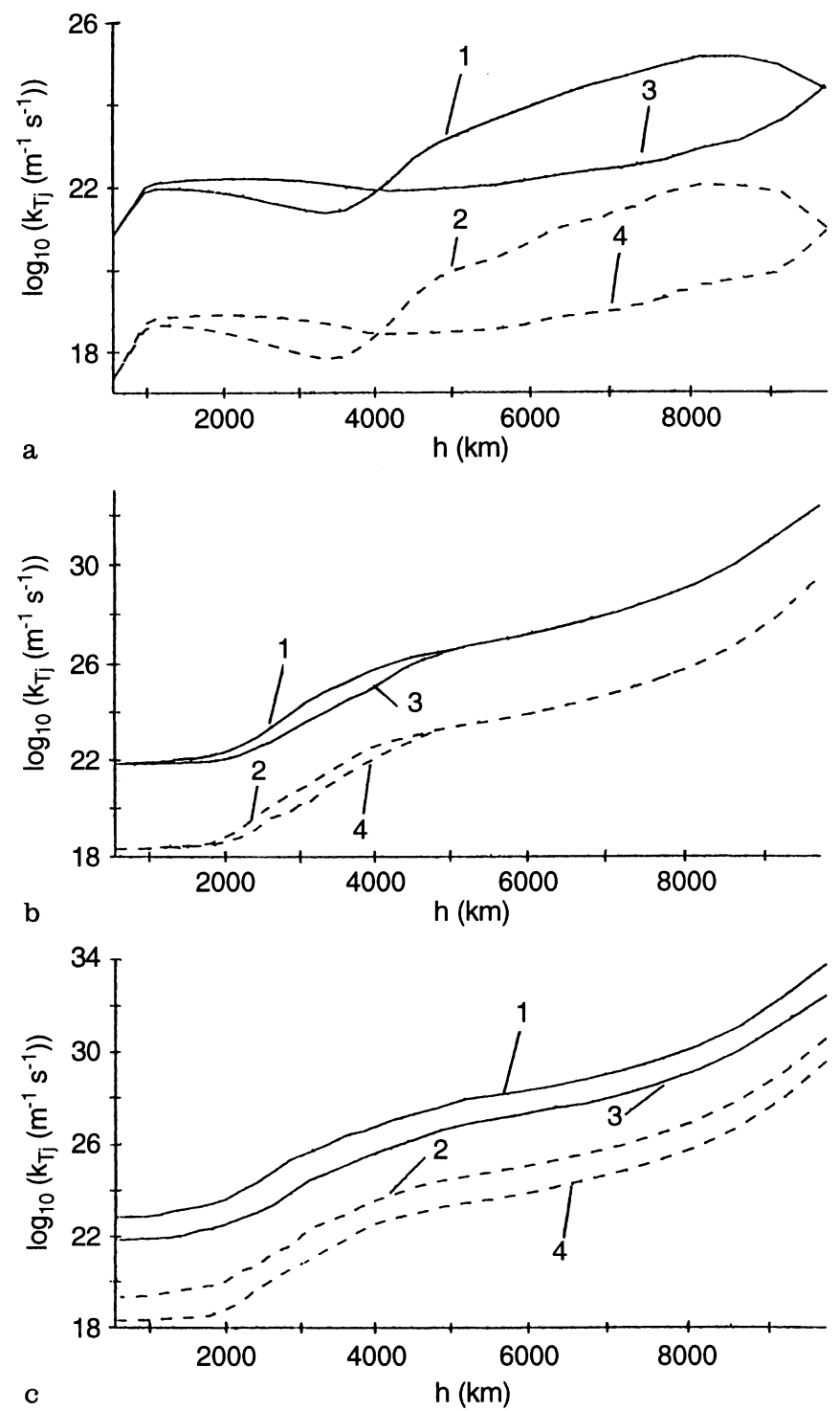

Fig. 3a-c. Coefficients of thermal conductivity $k_{T j}$ of protons $(j=1$, dashed curves) and electrons ( $j=2$, solid curves) versus altitude $h$. In a it is $j_{2}=10^{-6} \mathrm{~A} / \mathrm{m}^{2}, W /\left(n_{2} k_{B} T_{2}\right)=10^{-4}$ at $h=h_{m}$. In b $j_{2}=10^{-5} \mathrm{~A} / \mathrm{m}^{2}, W /\left(n_{2} k_{B} T_{2}\right)=10^{-3}$ at $h=h_{m}$. The indices $1,2(3$, 4) mean $C=1.5 \cdot 10^{-4} \mathrm{~s}^{-1}\left(C=1.5 \cdot 10^{-3} \mathrm{~s}^{-1}\right)$. In c, $j_{2}=10^{-5} \mathrm{~A} / \mathrm{m}^{2}$, $C=1.5 \cdot 10^{-3} \mathrm{~s}^{-1}$, the indices $1,2(3,4)$ represent $W /\left(n_{2} k_{B} T_{2}\right)=$ $10^{-4}\left(W /\left(n_{2} k_{B} T_{2}\right)=10^{-3}\right)$

with the altitude $h$. Comparing Fig. $2 \mathrm{a}$ and $2 \mathrm{~b}$ to each other, we conclude that this effect becomes more intensive as $j_{2}$ increases.

Then, Fig. 2c,d and (Fig. 2e, f) are presented for the electron (proton) temperature $T_{2}\left(T_{1}\right)$ in the turbulent region. Figure $2 \mathrm{c}$ and e (Fig. $2 \mathrm{~d}$ and $\mathrm{f}$ ) are presented for the case when the maximum value of $W /\left(n_{2} k_{B} T_{2}\right)$ is given as $1 \cdot 10^{-4}\left(1 \cdot 10^{-3}\right)$. These results are obtained for $j_{2}=1 \cdot 10^{-6} \mathrm{~A} / \mathrm{m}^{2}$. The curves marked with the indices 1, 2, 3, 4 and 5 in Fig. $2 \mathrm{c}$ are obtained for $C=1.5 \mathrm{~s}^{-1}, 1.5 \cdot 10^{-1} \mathrm{~s}^{-1}, 1.5 \cdot 10^{-2} \mathrm{~s}^{-1}, 1.5 \cdot 10^{-3} \mathrm{~s}^{-1}$, and $1.5 \cdot 10^{-4} \mathrm{~s}^{-1}$, respectively. These curves also correspond to the curves in Fig. $2 \mathrm{~d}-\mathrm{f}$. For instance, the minimum value $\left(C=1.5 \cdot 10^{-4} \mathrm{~s}^{-1}\right)$ may be found for $E_{\perp}=1 \mathrm{mV} / \mathrm{m}$ and $r_{\perp}=100 \mathrm{~km}$ at $h=1000 \mathrm{~km}$. In all the graphically presented cases, the approximations given to obtain Eqs. (18) and (19) are not valid.

Comparing Fig. 2c and e, we conclude that while $C$ decreases (see the curves 1-4) the electron temperature increases. This is so because the losses of the plasma energy from the turbulent flux tube due to the plasma convection become less effective. However, the increase of the electron temperature results in an increase of the thermal conductivity of the electron plasma. This effect prevents the electron temperature from a further increase. The coefficient of electron conductivity increases with the altitude $h$. Therefore, the maximum value of the electron temperature becomes less (see curve 5 in Fig. 2c) and takes place at lower values of the altitude $h$. The proton temperature also increases while $C$ decreases. However, this effect is weaker for the protons than for the electrons (see curves 1-4 in Fig. 2e). This is so because the friction force for the electrons forms a more intensive source of anomalous heating of the plasma than the proton friction force (see Eq. 9). On average, the coefficient of thermal conductivity takes lower values for the proton plasma than for the electron plasma. The main reason for this conclusion is that $m_{1} \gg m_{2}$. The weak thermal conductivity of the protons is not able to prevent the increase of the proton temperature (see curve 5 in Fig. 2e). The increase of the ratio $T_{1} / T_{2}$ results in a damping of the EIC turbulence because $\left|\gamma_{j}\right|$ decreases (Kindel and Kennel, 1971). Fig. 2d and f show the same effects as Fig. 2c and e. The more intensive turbulence results into a more intensive heating of the electron and proton plasma.

While the parameter $C$ decreases, the thermal conductivity influences more strongly the heat balance of the plasma in the turbulent region in comparison with the convection. Thus, it is important to study the behaviour of the coefficient of thermal conductivity when $C$ possesses smaller values. In Fig. 3, the solid (dashed) curves are shown for the electrons (protons). Figure $3 \mathrm{a}$ shows the case $j_{2}=1 \cdot 10^{-6} \mathrm{~A} / \mathrm{m}^{2}$, whilst Fig. $3 \mathrm{~b}$ and $3 \mathrm{c}$ are for $j_{2}=1 \cdot 10^{-5} \mathrm{~A} / \mathrm{m}^{2}$. In the case of Fig. 3a (3b), the maximum value is $W /\left(n_{2} k_{B} T_{2}\right)=$ $1 \cdot 10^{-4}\left(\mathrm{~W} /\left(n_{2} k_{B} T_{2}\right)=1 \cdot 10^{-3}\right)$. In Fig. 3a, b, the curves marked with the indices 1 and 2 ( 3 and 4 ) are shown for $C=1.5 \cdot 10^{-4} \mathrm{~s}^{-1}\left(C=1.5 \cdot 10^{-3} \mathrm{~s}^{-1}\right)$. To obtain Fig. 3c, we make $C=1.5 \cdot 10^{-3} \mathrm{~s}^{-1}$. Then, the curves marked with the indices 1 and 2 ( 3 and 4 ) are shown for the maximum value $W /\left(n_{2} k_{B} T_{2}\right)=1 \cdot 10^{-4}\left(1 \cdot 10^{-3}\right)$.

Analysis of the results presented in Fig. 3 shows the following: on average, $\lg \left(k_{T 2} / k_{T 1}\right) \sim(3-4)$. The increase of intensity of turbulence results, on average, in a decrease of the values of $k_{T j}$ (see Fig. 3c). The values of $k_{T j}$ increase with the altitude $h$. The smaller the values of $k_{T 1}$ the lower the effectiveness with which the anomalous thermal conductivity provides the heat transport from the magnetosphere into the ionosphere. This conclusion is especially important for the heat balance of the proton component of the plasma.

When the intensity of the parallel current increases and the turbulence is developed, generally the angle $\xi$ between $\mathbf{B}$ and $\mathbf{k}$ increases $(0 \leq \xi<\pi / 2)$. This results in a decrease of $v_{j}$ and an increase of $k_{T j}$. Comparing 

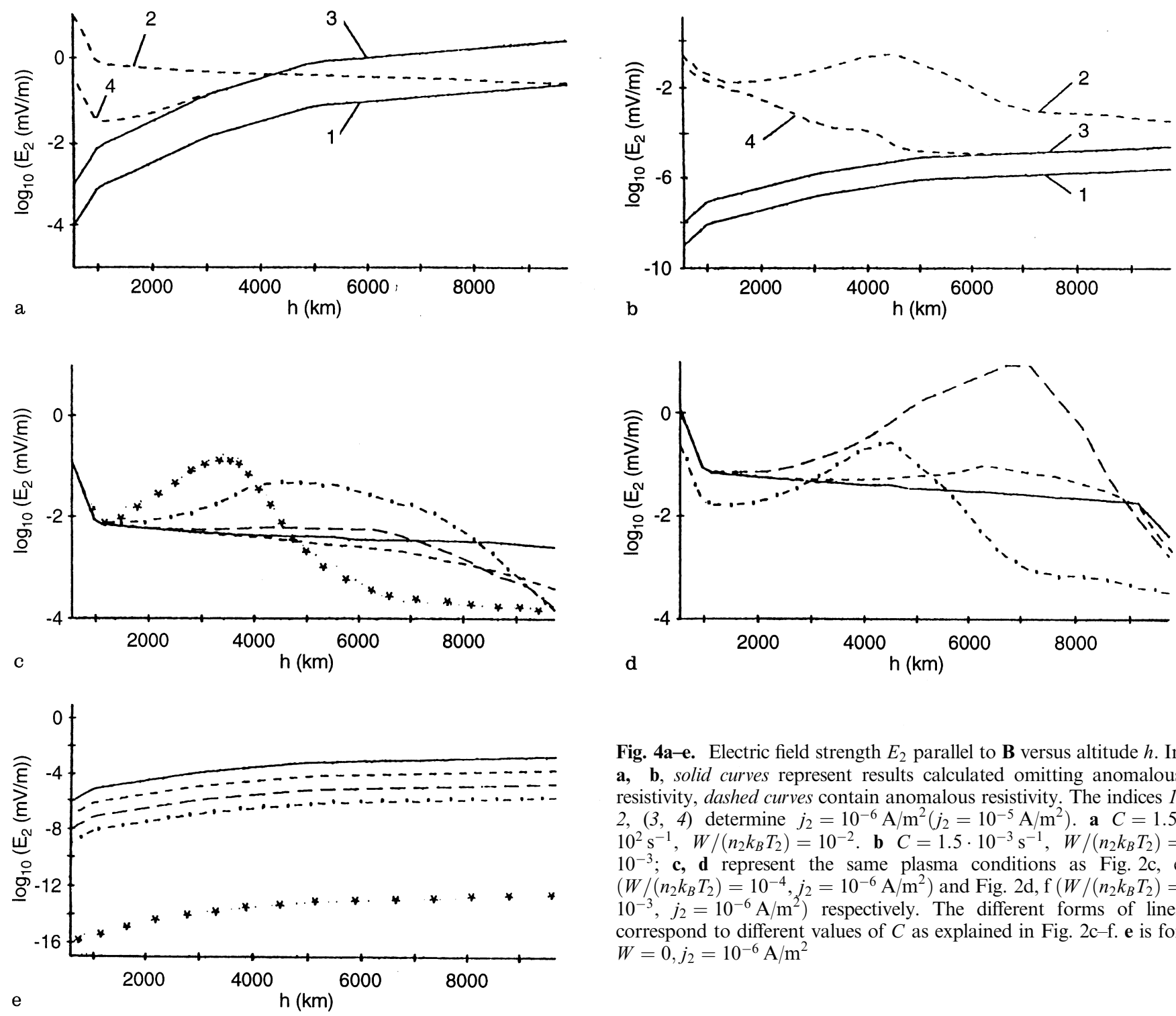

Fig. 4a-e. Electric field strength $E_{2}$ parallel to $\mathbf{B}$ versus altitude $h$. In a, b, solid curves represent results calculated omitting anomalous resistivity, dashed curves contain anomalous resistivity. The indices 1 , 2, $(3,4)$ determine $j_{2}=10^{-6} \mathrm{~A} / \mathrm{m}^{2}\left(j_{2}=10^{-5} \mathrm{~A} / \mathrm{m}^{2}\right)$. a $C=1.5$. $10^{2} \mathrm{~s}^{-1}, W /\left(n_{2} k_{B} T_{2}\right)=10^{-2}$. b $C=1.5 \cdot 10^{-3} \mathrm{~s}^{-1}, W /\left(n_{2} k_{B} T_{2}\right)=$ $10^{-3}$; c, d represent the same plasma conditions as Fig. $2 \mathrm{c}$, e $\left(W /\left(n_{2} k_{B} T_{2}\right)=10^{-4}, j_{2}=10^{-6} \mathrm{~A} / \mathrm{m}^{2}\right)$ and Fig. $2 \mathrm{~d}, \mathrm{f}\left(\mathrm{W} /\left(n_{2} k_{B} T_{2}\right)=\right.$ $\left.10^{-3}, j_{2}=10^{-6} \mathrm{~A} / \mathrm{m}^{2}\right)$ respectively. The different forms of lines correspond to different values of $C$ as explained in Fig. 2c-f. e is for $W=0, j_{2}=10^{-6} \mathrm{~A} / \mathrm{m}^{2}$

Figs. 3a and $\mathrm{b}$, we arrive at the same conclusion. The smaller values of $k_{T j}$ in the case of Fig. 3a in comparison with those of Fig. $3 \mathrm{~b}$ may not prevent from an effective heating of the plasma when $C$ decreases from $1.5 \cdot 10^{-3} \mathrm{~s}^{-1}$ to $1.5 \cdot 10^{-4} \mathrm{~s}^{-1}$. As shown in Fig. $2 \mathrm{c}$ (see curves 4 and 5), the heating leads to the increase (decrease) of the ratio $T_{1} / T_{2}$ at altitudes of $h>4000 \mathrm{~km}(h<4000 \mathrm{~km})$. Therefore, as shown in Fig. 3a, the values of $k_{T j}$ become greater (less) at altitudes of $h>4000 \mathrm{~km}(h<4000 \mathrm{~km})$ inside the turbulent region when $C$ decreases from $1.5 \cdot 10^{-3} \mathrm{~s}^{-1}$ to $1.5 \cdot 10^{-4} \mathrm{~s}^{-1}$. The same conclusions may be inferred from the results presented in Fig. 3b. It should be mentioned that the effect of the increase of $k_{T j}$ at altitudes of $h>4000 \mathrm{~km}$ is much weaker for Fig. $3 \mathrm{~b}$ than for Fig. 3a.

Let us now discuss the results of the calculations presented in Fig. 4. They are obtained for the parallel component $E_{2}$ of the electric field. We also calculated the total difference of the electric potential $\Delta V$. The latter is

gained along $\mathbf{B}$ at altitudes of $h_{b}<h<h_{m}$ in the flux tube given already. In Fig. $4 \mathrm{a}$, the solid curves are obtained using Eq. (18). In this case, the effects of anomalous resistivity are not taken into account. The dashed curves are obtained for the total values of $E_{2}$. The curves marked with the indices 1 and 2 (3 and 4) are shown for the same conditions as in the case of Fig. 2a (and b). We conclude that the effects of anomalous resistivity contribute most effectively to the values of $E_{2}$ at lower altitudes. In accordance with the behaviour of $v_{j}$ estimated already, this contribution is greater when $j_{2}=1 \cdot 10^{-6} \mathrm{~A} / \mathrm{m}^{2}$ than when $j_{2}=1 \cdot 10^{-5} \mathrm{~A} / \mathrm{m}^{2}$. In the cases of curves 1 and 2 ( 3 and 4) shown in Fig. 4a, we find $\Delta V$ to be about $0.8 \mathrm{kV}$ and $5.8 \mathrm{kV}(8.4 \mathrm{kV}$ and $8.5 \mathrm{kV}$ ), respectively.

The curves 1-4 shown in Fig. $4 \mathrm{~b}$ are found in analogy to the curves 1-4 of Fig. 4a. But for Fig. $4 \mathrm{~b}, C=1.5$. $10^{-3} \mathrm{~s}^{-1}$ and the maximum value $W /\left(n_{2} k_{B} T_{2}\right)=1 \cdot 10^{-3}$ rather than $C=1.5 \cdot 10^{2} \mathrm{~s}^{-1}$ and $W /\left(n_{2} k_{B} T_{2}\right)=1 \cdot 10^{-2}$ in Fig. 4a. Comparing Fig. 4a with Fig. 4b, one can 
make the following conclusions. While the parameter $C$ decreases the effects of anomalous resistivity contribute much more effectively to the values of $E_{2}$ at all altitudes $\left(h_{b}<h<h_{m}\right)$. On the other hand, under these conditions, the effects of the inertial force mentioned become negligible at the same altitudes excluding, perhaps, the high-altitude part of the turbulent region. In the cases of the curves 1 and 3 (2 and 4) shown in Fig. 4b, we estimate $\Delta V$ to be negligible (about $0.5 \mathrm{kV}$ and $0.1 \mathrm{kV}$ ), respectively.

Figure 4c (Fig. 4d) is presented for the same conditions as Fig. 2c, e (Fig. 2d, f). The curves shown in Fig $4 \mathrm{c}, \mathrm{d}$ may be compared with the curves shown in Fig. $2 \mathrm{~d}-\mathrm{f}$. Figure $4 \mathrm{e}$ is obtained for the same conditions as Fig. $4 c, d$, but the effects of anomalous resistivity are excluded using Eq. (18). Comparing the results of our calculations presented in Figs. 2 and 4, one can make the following conclusions: the strength of the parallel electric field is very sensitive to the changes of the proton and electron temperatures. In particular, the increase of the ratio $T_{1} / T_{2}$ results in a decrease of the friction force and the strength of the parallel electric field. Thus, the decrease of this ratio may result in an increase of $\Delta V$. For instance, the maximum value of $\Delta V$ obtained in Fig. $4 \mathrm{~d}$ amounts to about $15.5 \mathrm{kV}$. Comparing Fig. $4 \mathrm{c}$ and d, we conclude that generally the values of $E_{2}$ are greater when the intensity of turbulence is stronger. It should be noted that these results are obtained when the current, and not the voltage, source is given for the parallel electric fields.

\section{Conclusions}

The behaviour of the plasma and EIC waves was investigated for different scales $r_{\perp}$ at which intensive parallel currents may occur in the auroral magnetosphere. This was done in dependence on the intensity of the magnetospheric convection and the parallel currents. It is shown that, on the order of magnitude, the total difference $\Delta V$ of the electric potential along $\mathbf{B}$ may be as large as $1-10 \mathrm{kV}$. Under the same conditions, the proton and electron temperatures of the turbulent plasma are only a few times larger than those of the background plasma. The magnetospheric convection and the thermal conductivity of the plasma are considered to estimate the energy losses in the turbulent region.

Our numerical estimates of the values of $\Delta V$ and $E_{2}$ are in accordance with those predicted experimentally by Mozer (1976) and Reiff et al. (1988, 1993). The numerical estimates of the values of $v_{j}$ are in accordance with those obtained earlier by Liperovsky and Pudovkin (1983).

It is presumed that, first, intensive electric currents may develop in narrow flux tubes. As shown, the currents result, particularly, in the appearance of friction and inertial forces acting on the plasma. Where the magnetospheric convection is intensive, the inertial force approximately balances the electric force along $\mathbf{B}$. Under such conditions, $\Delta V$ may be as large as $1-$ $10 \mathrm{kV}$. The proton temperature has values close to its background values. In contrast, the electron temperature values are about one or two orders of magnitude greater than the corresponding background values. In this case ion acoustic turbulence may develop in the plasma.

While the model parameter $C\left(C=\Delta V_{\perp} / \Phi\right)$ decreases in value the electron temperature increases. This fact results in an increase of the thermal conductivity of the electron plasma. Further, this effect prevents the electron temperature from a further increase. The proton temperature also increases and, first, the ratio $T_{1} / T_{2}$ decreases while $C$ decreases. This is because the friction force forms a more intensive source of anomalous heating for the electron component of the plasma than for the proton component. Then, the thermal conductivity of the protons cannot compensate for the large increase of the proton temperature. We found $\lg \left(k_{T 2} / k_{T 1}\right) \sim(3-4)$. The increase of the ratio $T_{1} / T_{2}$ results in a damping of the EIC turbulence since $\left|\gamma_{j}\right|$ decreases (Kindel and Kennel, 1971). The more intensive turbulence results in a more intensive heating of the electron and proton plasma and in a decrease of the value of $k_{T j}$.

Let the intensity of the parallel current increase (from $j_{2}=1 \cdot 10^{-6} \mathrm{~A} / \mathrm{m}^{2}$ to $j_{2}=1 \cdot 10^{-5} \mathrm{~A} / \mathrm{m}^{2}$ at $h=h_{b}$ ), and let the turbulence be developed. In this case, on average, the angle $\xi$ between $\mathbf{B}$ and $\mathbf{k}$ increases $(0 \leq \xi<\pi / 2)$. This results in a decrease of $v_{j}$ and an increase of $k_{T j}$.

While the parameter $C$ decreases, the effects of anomalous resistivity contribute much more effectively to the values of $E_{2}$ at all altitudes $\left(h_{b}<h<h_{m}\right)$. The strength of the parallel electric field is very sensitive to the changes of the proton and electron temperatures. The increase of the ratio $T_{1} / T_{2}$ results in a decrease of the friction force and of the strength of $E_{2}$. Thus, the decrease of this ratio may result in an increase of $\Delta V$. When the current and not the voltage-source is given, the values of $E_{2}$ are greater when the intensity of turbulence is stronger. Thus, it is important to develop our model further taking the closure of the parallel currents in the ionosphere and in the plasma sheet into account. This will allow more realistic initial conditions for the plasma temperature at the top of the turbulent region in comparison with those considered.

Acknowledgements. We appreciate fruitful discussions with M. I. Pudovkin. This work is supported by RFFI under grant N 96-05000066. V. E. Zakharov would like to thank the organization Deutsche Forschungsgemeinschaft for financing his stay at Potsdam Astrophysical Institute to work on the given topic under project 436 RUS 113/77/3. C.-V. Meister thanks the Ministerium für Wissenschaft, Forschung und Kuttur des Landes Brandenburg for financial support by the project 24/2599-041055-1997.

Topical Editor K.-H. Glassmeier thanks two referees for their help in evaluating the paper.

\section{References}

André, M., Waves and wave-particle interactions in the auroral region, J. Atmos. Solar-Terr. Phys., 99, 1687-1712, 1997.

André, M., H. Koskinen, G. Gustafsson, and R. Lundin, Ion waves and upgoing ion beams observed by the Viking satellite, Geophys. Res. Lett., 14, 463-466, 1987. 
Artsymovich, L. A., and R. Z. Sagdeev, Plasma Physics for Physicists (in Russian), Atomizdat, Moscow, 1979.

Chiu, T. T., and J. M. Cornwall, Electrostatic model of a quiet auroral arc, J. Geophys. Res., 85, 543-556, 1980.

Chust, Tr., P. Louarn, M. Volwerk, H. de Feraudy, J.-E. Wahlund, and B. Holback, Electric fields with a large parallel component observed by the Freja spacecraft: artifacts or real signals?, J. Geophys. Res., 103, 215-224, 1998.

Erlandson, R. E., L. J. Zanetti, M. H. Acuña, A. I. Eriksson, L. Eliasson, M. H. Boehm, and L. G. Blomberg, Freja observations of electromagnetic ion cyclotron ELF waves and transverse oxygen ion acceleration on auroral field lines, Geophys. Res. Lett., 21, 1855-1858, 1994.

Evans, D. S., Precipitating electron fluxes formed by magnetic fieldaligned potential difference, J. Geophys. Res., 79, 2853-2858, 1974.

Fatkullin, M. N., and Y. S. Sitnov, A dipolar coordinate system and some of its characteristics, Geomagn. Aeronomy (in Russian), 12, 333-335, 1972.

Foster, J. C., J. M. Holt, R. G. Musgrove, and D. S. Evans, Ionospheric convection associated with discrete levels of particle precipitation, Geophys. Res. Lett., 13, 656-659, 1986.

Goldstone, R. J., and P. H. Rutherford, Introduction to Plasma Physics, Institute of Physics Publishing, Bristol, 1995.

Gorney, D. J., Potential structures and particle acceleration on auroral field lines, Adv. Space Res., 4, 499-508, 1984.

Gustafsson, G., M. André, L. Matson, and H. Koskinen, On waves below the local proton gyrofrequency in auroral acceleration regions, J. Geophys. Res., 95, 5889-5603, 1990.

Heppner, J. P., and N. C. Maynard, Empirical high-latitude electric field models, J. Geophys. Res., 92, 4467-4489, 1987.

Holt, J. M., R. H. Wand, D. S. Evans, and W. L. Oliver, Empirical models for the plasma convection at high latitudes from Millstone Hill observations, J. Geophys. Res., 92, 203-212, 1987.

Hudson, M. K., R. L. Lysak, and F. S. Mozer, Magnetic fieldaligned potential drops due to electrostatic ion cyclotron turbulence, Geophys. Res. Lett., 5. 143-146, 1978.

Iijima, T., and T. A, Potemra, Large-scale characteristics of fieldaligned currents associated with substorms, J. Geophys. Res., 83, 599-615, 1978.

Kalitkin, N. N., Numerical Methods (in Russian), Nauka, Moscow, 1978.
Kindel, J. M., and C. F. Kennel, Topside current instabilities, J. Geophys. Res., 76, 3055-3078, 1971.

Liperovsky, V. A., and M. I. Pudovkin, Anomalous Resistivity and Double Layers in the Magnetospheric Plasma (in Russian), Nauka, Moscow, 1983.

Lominadze, D. G., and K. N. Stepanov, Excitation of the lowfrequency longitudinal oscillations in the magnetizied plasma, J. Theor. Phys. (in Russian), 34, 1823-1634, 1964.

Lund, E. J., and J. LaBelle, On the generation and propagation of auroral electromagnetic ion cyclotron waves, J. Geophys. Res., 102, 17241-17253, 1997.

Lysak. R. L., and T. Dum, Dynamics of magnetosphere-ionosphere coupling including turbulent transport. J. Geophys. Res., 88, 365-380, 1983.

Mozer, F. S., Observations of large parallel electric fields in the auroral ionosphere, Ann. Geophysicae, 32, 97-107, 1976.

Oscarsson, T., A. Vaivads, K. Rönnmark, J. H. Clemmons, H. de Feraudy, and B. Holback, Toward a consistent picture of the generation of electromagnetic ion cyclotron ELF waves on auroral field lines. J. Geophys. Res., 102, 24369-24386, 1997.

Reiff, P. H., H. L. Collin, J. D. Craven, J. L. Burch, J. D. Winningham, E. G. Shelley, L. A. Frank, and M. A. Friedman, Determination of auroral electrostatic potentials using highand low-altitude particle distributions, J. Geophys. Res., 93, 7441-7465, 1988.

Reiff P. H., G. Lu, J. L. Burch, J. D. Winningham, L. A. Frank, J. D. Craven, W. K. Peterson, and R. A. Heelis, On the high- and low- altitude limits of the auroral electric field region, in Auroral plasma dynamics, Eds. R. L. Lysak, AGU, Geophys. Monogr. 80, Washington, 143-154, 1993.

Samarsky, A. A., and E. C. Nikolaev, The Integration Methods for the Grid Equations (in Russian), Nauka, Moscow, 1978.

Senior, C., D. Fontaine, and G. Caudal, Convection electric fields and electrostatic potential over $61^{\circ}<\Lambda<72^{\circ}$ invariant latitude observed with European incoherent scatter facility, 2, Statistical results, Ann. Geophysicae, 8, 257-272, 1990.

Temerin, M., C. Carlson, and J. P. McFadden, The acceleration of electrons by electromagnetic ion cyclotron waves, in Auroral plasma dynamics, Eds. R. L. Lysak, AGU, Geophys. Monogr. 80., Washington, 155-167, 1993.

Torbert, R. B., and F. S. Mozer, Electrostatic shocks as the source of discrete auroral arcs, Geophys. Res. Lett., 5, 135-138, 1978. 